\title{
Polarization transfer via field sweeping in parahydrogen-enhanced nuclear magnetic resonance
}

\author{
James Eills, ${ }^{1,2, a)}$ John W. Blanchard, ${ }^{2}$ Teng Wu, ${ }^{2}$ Christian Bengs, ${ }^{1}$ Julia Hollenbach, ${ }^{1}$ Dmitry Budker, ${ }^{2,3}$ and \\ Malcolm H. Levitt ${ }^{1}$ \\ 1) University of Southampton, Southampton, United Kingdom \\ ${ }^{2)}$ Helmholtz Institute, Johannes-Gutenberg University, Mainz, Germany \\ 3) University of California, Berkeley, California, USA
}

(Dated: 1 April 2019)

We show that in a spin system of two magnetically inequivalent protons coupled to a heteronucleus such as ${ }^{13} \mathrm{C}$, an adiabatic magnetic field sweep, passing through zero field, transfers proton singlet order into magnetization of the coupled heteronucleus. This effect is potentially useful in parahydrogen-enhanced nuclear magnetic resonance, and is demonstrated on singlet-hyperpolarized $\left[1-{ }^{13} \mathrm{C}\right]$ maleic acid, which is prepared via the reaction between $\left[1-{ }^{13} \mathrm{C}\right]$ acetylene dicarboxylic acid and para-enriched hydrogen gas. The magnetic field sweeps are of microtesla amplitudes, and have durations on the order of seconds. We show a polarization enhancement by a factor of $10^{4}$ in the ${ }^{13} \mathrm{C}$ spectra of $\left[1-{ }^{13} \mathrm{C}\right]$ maleic acid in a $1.4 \mathrm{~T}$ magnetic field.

Keywords: NMR, Hyperpolarization, Adiabaticity, Zero-field NMR

\section{INTRODUCTION}

Magnetic resonance spectroscopy and magnetic resonance imaging are widely applied techniques, but are often limited by the very low polarization of nuclear spins at ordinary temperatures even in the highest available magnetic fields. Nuclear hyperpolarization techniques overcome this limitation by strongly polarizing the nuclear spins, with polarization levels of several percent regularly achieved ${ }^{1-4}$.

One hyperpolarization method is parahydrogeninduced polarization (PHIP) which engenders nuclear spin polarization from the nuclear spin-singlet $(I=0)$ para isomer of hydrogen gas ${ }^{5-9}$. In hydrogenative PHIP, a molecule of para-enriched hydrogen undergoes pairwise addition to a substrate molecule. If the protons in the product retain magnetic equivalence (i.e. the Hamiltonian is invarant with respect to exchange of the proton spins), the hyperpolarized singlet state population remains locked in an unobservable form. If their magnetic equivalence is strongly broken (with respect to the magnitude of the proton-proton $J$-coupling), as is the case for the original demonstrations ${ }^{5}$, the hyperpolarized magnetization may be directly observed. However, if the breaking of magnetic equivalence is only slight, as is the case in this work, magnetic field or pulse manipulations are needed to convert the hyperpolarized singlet order into magnetization ${ }^{10-33}$. The breaking of magnetic equivalence may be through a chemical shift difference, or as in our case, asymmetric coupling to a third nucleus. Transfer of the parahydrogen proton spin polarization to heteronuclear magnetization may be achieved by using high-field rf (radio frequency) pulses ${ }^{11-22}$, hydrogenating at ultra-low field matching conditions ${ }^{23-26}$, or by magnetic field cycling techniques involving a sudden jump

\footnotetext{
a) Electronic mail: eills@soton.ac.uk
}

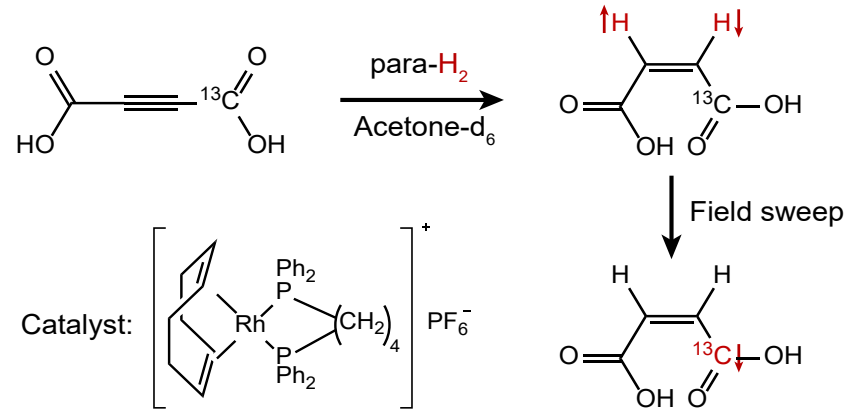

FIG. 1. a) Para-enriched hydrogen gas undergoes pairwise addition to $\left[1-{ }^{13} \mathrm{C}\right]$ acetylene dicarboxylic acid to form $\left[1-{ }^{13} \mathrm{C}\right]$ maleic acid. The reaction catalyst is [1,4-bis(diphenylphosphino)butane $](1,5-$ cyclooctadiene)rhodium(I) tetrafluoroborate. The proton singlet order is transformed into ${ }^{13} \mathrm{C}$ magnetization by applying a field sweep, passing through zero field.

in the magnetic field to a low value followed by a slow increase in the field ${ }^{27-31}$.

We demonstrate an alternative field-cycling method in which proton singlet order is efficiently converted into heteronuclear magnetization by adiabatically sweeping an applied magnetic field from a negative value to a positive value, passing through zero. In this work we study the $2 \%$ natural abundance $\left[1-{ }^{13} \mathrm{C}\right]$ maleic acid molecules formed after hydrogenating acetylene dicarboxylic acid (see Fig. 1). The method is depicted in Fig. 2: (1) react $\left[1-{ }^{13} \mathrm{C}\right]$ acetylene dicarboxylic acid with para-enriched hydrogen in the presence of a magnetic field (magnetic field $=+2 \mu \mathrm{T})$; (2) perform a non-adiabatic (rapid) field reversal to $-2 \mu \mathrm{T} ;(3)$ adiabatically (slowly) reverse the sign of the magnetic field, passing through zero; (4) shuttle the sample to a high-field benchtop NMR (nuclear magnetic resonance) magnet, and (5) observe the ${ }^{13} \mathrm{C}$ NMR signals. To perform the field sweeps, we employ a ZULF 


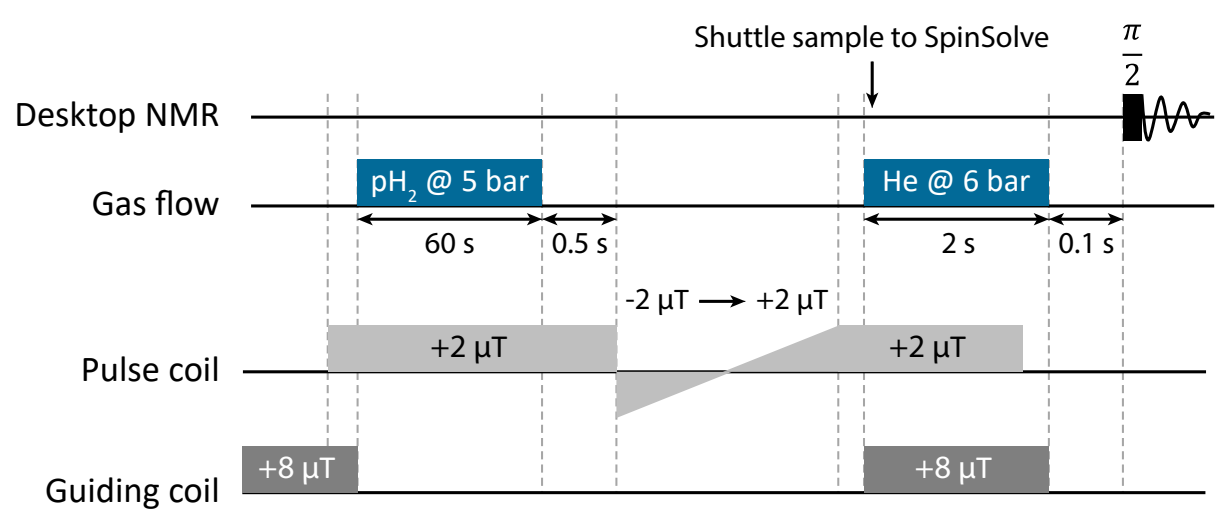

FIG. 2. Event sequence for the experiments performed, unless stated otherwise in the text. Further details are given in the Materials and Methods section.

(zero and ultra-low field) NMR setup ${ }^{34}$, which allows for precise control over the magnetic fields required for polarization transfer. The apparatus is described in more detail in the Materials and Methods section. Adiabatic field sweeps passing through zero have also been used in the context of chemically induced dynamic nuclear polarization (CIDNP) ${ }^{35}$.

$\left[1-{ }^{13} \mathrm{C}\right]$ maleic acid is a 3 -spin- $1 / 2, \quad \mathrm{AA}^{\prime} \mathrm{X}$ system. These types of systems have been studied in the context of SABRE (signal amplification by reversible exchange) ${ }^{32,33}$. The $J$-coupling network is shown in Fig. 3a, along with experimental (Fig. 3b) and simulated (Fig. 3c) ${ }^{13} \mathrm{C}$ NMR spectra for a system at thermal equilibrium. The proton-proton $J$-coupling is of the same order of magnitude as the difference in proton-carbon $J$ couplings, so the proton singlet state, which is populated from parahydrogen addition, is close to being an eigenstate of the spin Hamiltonian. An energy level diagram with the single-quantum ${ }^{13} \mathrm{C}$ transitions highlighted is shown in Fig. 3d, to illustrate the origin of the spectral lines. The finite amplitude of the outer transitions (5) and (6) is due to singlet-triplet mixing, induced by the heteronuclear $J$-couplings.

\section{THEORY}

\section{A. State Bases and Hamiltonians}

In this article we study three-spin systems, with two protons, $I_{1}$ and $I_{2}$, from the parahydrogen pair, and a heteronuclear ${ }^{13} \mathrm{C}$ spin, $S_{3}$. It is convenient to discuss the dynamics of this spin system using a set of orthogonal basis states termed here the $\mathbf{S T Z}$ (Singlet-Triplet-Zeeman) basis, defined as the tensor product of the singlet-triplet states for spins 1 and 2, and the Zeeman states for spin 3 :

$$
\mathbf{S T Z}=\left\{\left|S_{0}^{12}\right\rangle,\left|T_{+1}^{12}\right\rangle,\left|T_{0}^{12}\right\rangle,\left|T_{-1}^{12}\right\rangle\right\} \otimes\left\{\left|\alpha^{3}\right\rangle,\left|\beta^{3}\right\rangle\right\}
$$

where the singlet and triplet states of the proton pair are defined as follows:

$$
\begin{aligned}
\left|S_{0}^{12}\right\rangle & =\frac{1}{\sqrt{2}}\left(\left|\alpha^{1} \beta^{2}\right\rangle-\left|\beta^{1} \alpha^{2}\right\rangle\right) \\
\left|T_{+1}^{12}\right\rangle & =\left|\alpha^{1} \alpha^{2}\right\rangle \\
\left|T_{0}^{12}\right\rangle & =\frac{1}{\sqrt{2}}\left(\left|\alpha^{1} \beta^{2}\right\rangle+\left|\beta^{1} \alpha^{2}\right\rangle\right) \\
\left|T_{-1}^{12}\right\rangle & =\left|\beta^{1} \beta^{2}\right\rangle .
\end{aligned}
$$

$|\alpha\rangle$ and $|\beta\rangle$ are the Zeeman spin states for an isolated spin in a magnetic field, with angular-momentum projection $+1 / 2$ and $-1 / 2$ along the external field axis (defined here as $z$ ), i.e. $I_{z}|\alpha\rangle=+\frac{1}{2}|\alpha\rangle$, and $I_{z}|\beta\rangle=-\frac{1}{2}|\beta\rangle$, where $\hbar=1$. The indices indicate the spin label.

For example, the state $\left|S_{0} \alpha\right\rangle=\frac{1}{\sqrt{2}}(|\alpha \beta \alpha\rangle-|\beta \alpha \alpha\rangle)$ signifies a proton singlet state, with the third spin $\left({ }^{13} \mathrm{C}\right.$ in the case here) being in the $|\alpha\rangle$ Zeeman state. The spin labels will be dropped henceforth.

The spin Hamiltonian for a solution-state sample is

$$
H=H_{0}+H_{1}
$$

which is split into two parts for convenience (discussed below), with

$$
\begin{gathered}
H_{0}=2 \pi J_{\mathrm{II}} \mathbf{I}_{1} \cdot \mathbf{I}_{2}+2 \pi J_{13} I_{1 z} S_{3 z}+2 \pi J_{23} I_{2 z} S_{3 z} \\
+\omega_{\mathrm{I}}^{0}\left(I_{1 z}+I_{2 z}\right)+\omega_{\mathrm{S}}^{0} S_{3 z}
\end{gathered}
$$

and

$$
\begin{aligned}
& H_{1}=2 \pi J_{13} \frac{1}{2}\left(I_{1}^{+} S_{3}^{-}+I_{1}^{-} S_{3}^{+}\right) \\
& \quad+2 \pi J_{23} \frac{1}{2}\left(I_{2}^{+} S_{3}^{-}+I_{2}^{-} S_{3}^{+}\right),
\end{aligned}
$$

where $\omega_{j}^{0}=-\gamma_{j} B$, with $j$ a spin label that corresponds to a specific nuclear isotope, and $J_{13}$ is the $J$-coupling between spins $I_{1}$ and $S_{3}$. The following symbols are introduced now for brevity in later discussions:

$$
\begin{aligned}
\omega_{\mathrm{II}} & =2 \pi J_{\mathrm{II}} \\
\omega_{\mathrm{IS}}^{\Delta} & =2 \pi\left(J_{13}-J_{23}\right) \\
\omega_{\mathrm{IS}}^{\Sigma} & =2 \pi\left(J_{13}+J_{23}\right) \\
\Omega & =\sqrt{4\left(\omega_{\mathrm{II}}\right)^{2}+\left(\omega_{\mathrm{IS}}^{\Delta}\right)^{2}} .
\end{aligned}
$$


a
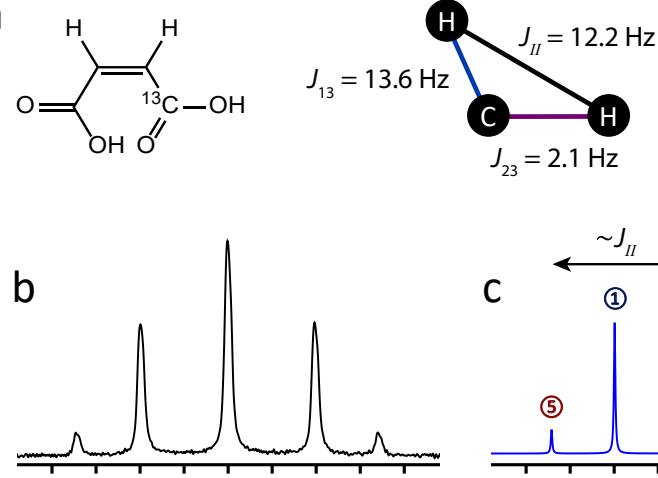

${ }^{13} \mathrm{C}$ Chemical Shift

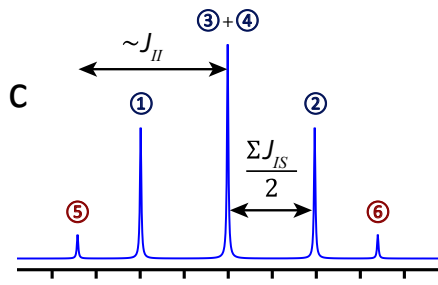

${ }^{13} \mathrm{C}$ Chemical Shift

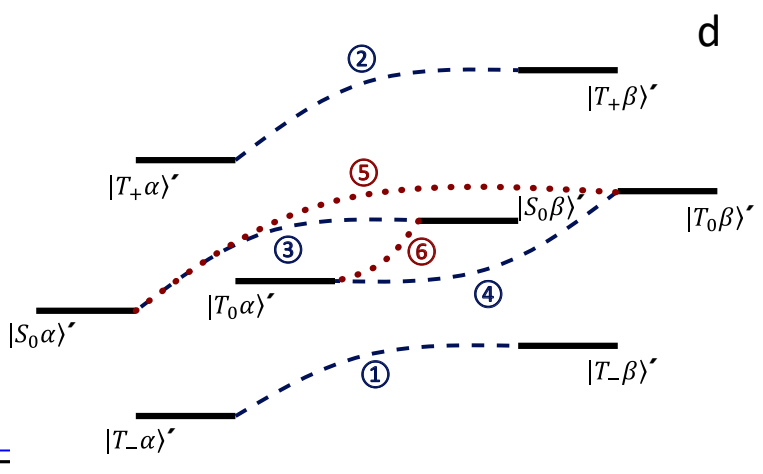

d

FIG. 3. a) The product of the hydrogenation reaction, $\left[1-{ }^{13} \mathrm{C}\right]$ maleic acid, alongside a schematic showing the $J$-coupling network. b) An experimental ${ }^{13} \mathrm{C}$ NMR spectrum without proton decoupling acquired with 16 transients on a sample of $1 \mathrm{M}$ maleic acid in methanol- $\mathrm{d}_{4}$, showing just the carbonyl ${ }^{13} \mathrm{C}$ peak. c) A simulation of the thermal polarization ${ }^{13} \mathrm{C}$ NMR spectrum of the carbonyl peak in $\left[1-{ }^{13} \mathrm{C}\right]$ maleic acid. d) The nuclear spin energy levels of $\left[1-{ }^{13} \mathrm{C}\right]$ maleic acid with the ${ }^{13} \mathrm{C}$ single quantum transitions labelled. The label on each state indicates the STZ basis state which corresponds most closely to the energy eigenstate. The dotted red lines labelled $(5)+(6)$ show weak transitions which would be rigorously forbidden if the singlet and triplet states were exact Hamiltonian eigenstates, but become weakly allowed through singlet-triplet mixing by the heteronuclear $J$-couplings.

The eigenbasis of $H_{0}$ is termed here the $\mathbf{S T Z} \mathbf{Z}^{\prime}$ basis, and consists of the following states:

$$
\begin{aligned}
& |1\rangle=\left|S_{0} \alpha\right\rangle^{\prime}=\cos \left(\frac{\theta_{\mathrm{G}}}{2}\right)|\beta \alpha \alpha\rangle-\sin \left(\frac{\theta_{\mathrm{G}}}{2}\right)|\alpha \beta \alpha\rangle \\
& |2\rangle=\left|T_{+1} \alpha\right\rangle^{\prime}=|\alpha \alpha \alpha\rangle \\
& |3\rangle=\left|T_{0} \alpha\right\rangle^{\prime}=\cos \left(\frac{\theta_{\mathrm{G}}}{2}\right)|\alpha \beta \alpha\rangle+\sin \left(\frac{\theta_{\mathrm{G}}}{2}\right)|\beta \alpha \alpha\rangle \\
& |4\rangle=\left|T_{-1} \alpha\right\rangle^{\prime}=|\beta \beta \alpha\rangle \\
& |5\rangle=\left|S_{0} \beta\right\rangle^{\prime}=\cos \left(\frac{\theta_{\mathrm{G}}}{2}\right)|\alpha \beta \beta\rangle-\sin \left(\frac{\theta_{\mathrm{G}}}{2}\right)|\beta \alpha \beta\rangle \\
& |6\rangle=\left|T_{+1} \beta\right\rangle^{\prime}=|\alpha \alpha \beta\rangle \\
& |7\rangle=\left|T_{0} \beta\right\rangle^{\prime}=\cos \left(\frac{\theta_{\mathrm{G}}}{2}\right)|\beta \alpha \beta\rangle+\sin \left(\frac{\theta_{\mathrm{G}}}{2}\right)|\alpha \beta \beta\rangle \\
& |8\rangle=\left|T_{-1} \beta\right\rangle^{\prime}=|\beta \beta \beta\rangle,
\end{aligned}
$$

where the 'Goldman angle' 13 is given by $\theta_{\mathrm{G}}=$ $\arctan \left(2 \omega_{\text {II }} / \omega_{\text {IS }}^{\Delta}\right)$. In the case of magnetic equivalence, $\theta_{\mathrm{G}}=\pi / 2$, while for strong inequivalence, $\theta_{\mathrm{G}} \rightarrow 0$. Note: some workers in the field use $\theta=\pi / 2-\theta_{\mathrm{G}}$.

The heteronuclear $J$-coupling terms in $H_{0}$ mix the $\left|S_{0}\right\rangle$ and $\left|T_{0}\right\rangle$ states. The eigenvalues for $H_{0}$ are given in Table I, along with the corresponding $\mathbf{S T} \mathbf{Z}^{\prime}$ eigenstate. Note that although the eigenvalues of $H_{0}$ depend on the applied field, the eigenstates do not.

\section{B. Avoided Crossings}

At specific matching conditions of the external field $B$, eigenvalues of $H_{0}$ can become degenerate. The flip-flop components of the heteronuclear $J$-couplings, contained in $H_{1}$, mix the basis states of the full Hamiltonian $H$,

\begin{tabular}{c||c} 
Eigenstate of $\boldsymbol{H}_{0}$ & $\mathbf{4} *$ State Energy $/ \mathbf{r a d ~ s}^{-\mathbf{1}}$ \\
\hline \hline$|1\rangle=\left|S_{0} \alpha\right\rangle^{\prime}$ & $-\omega_{\mathrm{II}}-\Omega+2 \omega_{\mathrm{S}}^{0}$ \\
\hline$|2\rangle=\left|T_{+1} \alpha\right\rangle^{\prime}$ & $\omega_{\mathrm{II}}+\omega_{\mathrm{IS}}^{\Sigma}+4 \omega_{\mathrm{I}}^{0}+2 \omega_{\mathrm{S}}^{0}$ \\
\hline$|3\rangle=\left|T_{0} \alpha\right\rangle^{\prime}$ & $-\omega_{\mathrm{II}}+\Omega+2 \omega_{\mathrm{S}}^{0}$ \\
\hline$|4\rangle=\left|T_{-1} \alpha\right\rangle^{\prime}$ & $\omega_{\mathrm{II}}-\omega_{\mathrm{IS}}^{\Sigma}-4 \omega_{\mathrm{I}}^{0}+2 \omega_{\mathrm{S}}^{0}$ \\
\hline$|5\rangle=\left|S_{0} \beta\right\rangle^{\prime}$ & $-\omega_{\mathrm{II}}-\Omega-2 \omega_{\mathrm{S}}^{0}$ \\
\hline$|6\rangle=\left|T_{+1} \beta\right\rangle^{\prime}$ & $\omega_{\mathrm{II}}-\omega_{\mathrm{IS}}^{\Sigma}+4 \omega_{\mathrm{I}}^{0}-2 \omega_{\mathrm{S}}^{0}$ \\
\hline$|7\rangle=\left|T_{0} \beta\right\rangle^{\prime}$ & $-\omega_{\mathrm{II}}+\Omega-2 \omega_{\mathrm{S}}^{0}$ \\
\hline$|8\rangle=\left|T_{-1} \beta\right\rangle^{\prime}$ & $\omega_{\mathrm{II}}+\omega_{\mathrm{IS}}^{\Sigma}-4 \omega_{\mathrm{I}}^{0}-2 \omega_{\mathrm{S}}^{0}$
\end{tabular}

TABLE I. The symbols are defined as follows: $\omega_{j}^{0}=-\gamma_{j} B$, and $\Omega=\sqrt{4\left(\omega_{\mathrm{II}}\right)^{2}+\left(\omega_{\mathrm{IS}}^{\Delta}\right)^{2}}$.

which leads to avoided crossings between the mixed states at the positions of the $H_{0}$ degeneracies. The $B$ field positions of these avoided crossings are given (in units of tesla) by:

$$
B_{\mathrm{AC}}^{(1)} \rightarrow \frac{2 \omega_{\mathrm{II}}+\Omega-\omega_{\mathrm{IS}}^{\Sigma}}{4\left(\gamma_{\mathrm{I}}-\gamma_{\mathrm{S}}\right)},
$$

and

$$
B_{\mathrm{AC}}^{(2)} \rightarrow \frac{\left.2 \omega_{\mathrm{II}}-\Omega-\omega_{\mathrm{IS}}^{\Sigma}\right)}{4\left(\gamma_{\mathrm{I}}-\gamma_{\mathrm{S}}\right)} .
$$



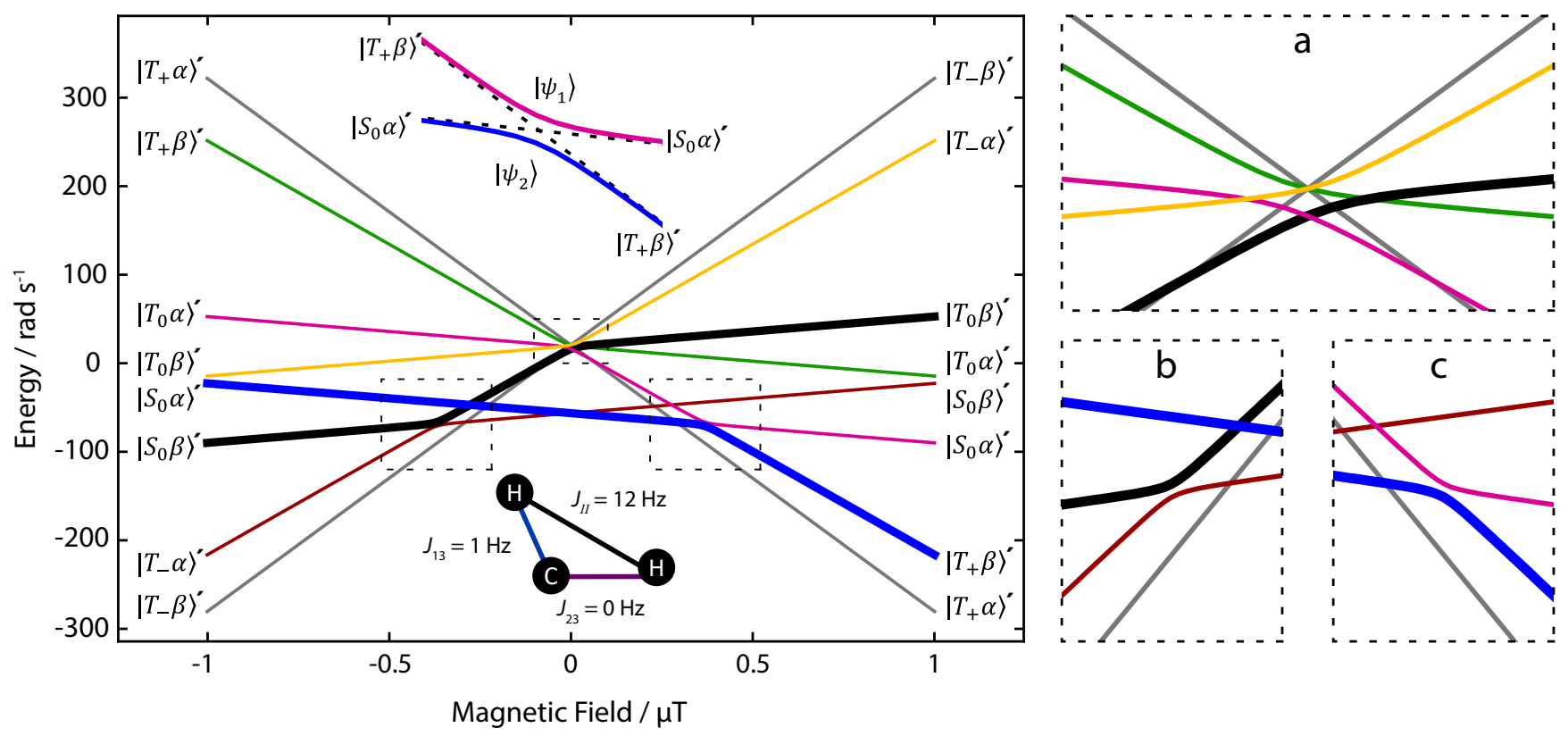

FIG. 4. Eigenvalues of the Hamiltonian $H$ for the model three-spin-1/2 system shown in the inset, as a function of applied field. There are four avoided crossings at $-369 \mathrm{nT},-8 \mathrm{nT},+8 \mathrm{nT}$, and $+369 \mathrm{nT}$ (shown enlarged in panels a, b, and c). The line colours label eigenvalues corresponding to eigenstates that transform smoothly into each other as the magnetic field changes. The thick black and blue lines indicate the evolution of singlet order during a (fully) adiabatic $-1 \rightarrow+1 \mu \mathrm{T}$ field sweep from proton $S_{0}$ polarization at $-1 \mu \mathrm{T}$ into ${ }^{13} \mathrm{C} \beta$ polarization at $+1 \mu \mathrm{T}$. The avoided crossing of a pair of Hamiltonian eigenvalues is shown in the inset. The corresponding eigenvalues of $H_{0}$ are shown by the dashed lines; the corresponding eigenstates of $H_{0}$ are termed the diabatic states and belong to the $\mathbf{S} \mathbf{T} \mathbf{Z}^{\prime}$ basis (Eqn. 6). The $H$ eigenstates are denoted $\left|\psi_{1}\right\rangle$ and $\left|\psi_{2}\right\rangle$. Further details are given in the text.

When $\left|\omega_{\text {II }}\right| \gg\left|\omega_{\text {IS }}^{\Delta}\right|$ these equations simplify to:

$$
B_{\mathrm{AC}}^{(1)} \rightarrow \frac{4 \omega_{\mathrm{II}}-\omega_{\mathrm{IS}}^{\Sigma}}{4\left(\gamma_{\mathrm{I}}-\gamma_{\mathrm{S}}\right)},
$$

and

$$
B_{\mathrm{AC}}^{(2)} \rightarrow \frac{-\omega_{\mathrm{IS}}^{\Sigma}}{4\left(\gamma_{\mathrm{I}}-\gamma_{\mathrm{S}}\right)}
$$

Table II shows the states which have an avoided crossing at the field values given, and gives the mixing terms in the Hamiltonian $H_{1}$ that cause these avoided crossings. The energy separation between the two states at the position of the avoided crossing is given by twice the magnitude of the mixing term.

The 8 eigenvalues of $H_{0}$ are plotted against the field strength $B$ in Fig. 4, for the case of a model spin system with $J_{\text {II }}=12 \mathrm{~Hz}, J_{13}=1 \mathrm{~Hz}$, and $J_{23}=0 \mathrm{~Hz}$. These values were chosen for clarity. $12 \mathrm{~Hz}$ is a typical $J$-coupling for two protons in a cis conformation across a double bond (as is often the case for parahydrogen products). The four avoided crossings in this system, connecting the states in Table II, are visible.

To understand the role of the avoided crossings, consider the inset in Fig. 4, which shows the avoided crossing between the $H_{0}$ eigenvalues corresponding to the diabatic

\begin{tabular}{c||c||c} 
Crossing states & Field & Mixing term \\
\hline \hline$\left|S_{0} \beta\right\rangle^{\prime} \leftrightarrow\left|T_{-1} \alpha\right\rangle^{\prime}$ & $-B_{\mathrm{AC}}^{(1)}$ & $\pi\left(J_{13} \cos \left(\frac{\theta_{\mathrm{G}}}{2}\right)-J_{23} \sin \left(\frac{\theta_{\mathrm{G}}}{2}\right)\right)$ \\
\hline$\left|S_{0} \alpha\right\rangle^{\prime} \leftrightarrow\left|T_{+1} \beta\right\rangle^{\prime}$ & $B_{\mathrm{AC}}^{(1)}$ & $\pi\left(J_{13} \cos \left(\frac{\theta_{\mathrm{G}}}{2}\right)-J_{23} \sin \left(\frac{\theta_{\mathrm{G}}}{2}\right)\right)$ \\
\hline$\left|T_{0} \beta\right\rangle^{\prime} \leftrightarrow\left|T_{-1} \alpha\right\rangle^{\prime}$ & $-B_{\mathrm{AC}}^{(2)}$ & $\pi\left(J_{13} \sin \left(\frac{\theta_{\mathrm{G}}}{2}\right)+J_{23} \cos \left(\frac{\theta_{\mathrm{G}}}{2}\right)\right)$ \\
\hline$\left|T_{0} \alpha\right\rangle^{\prime} \leftrightarrow\left|T_{+1} \beta\right\rangle^{\prime}$ & $B_{\mathrm{AC}}^{(2)}$ & $\pi\left(J_{13} \sin \left(\frac{\theta_{\mathrm{G}}}{2}\right)+J_{23} \cos \left(\frac{\theta_{\mathrm{G}}}{2}\right)\right)$
\end{tabular}

TABLE II.

states $\left|S_{0} \alpha\right\rangle^{\prime}$ and $\left|T_{+1} \beta\right\rangle^{\prime}$. The eigenvalues of the diabatic states (i.e. the eigenstates of $H_{0}$ ) are shown by dotted black lines, and the coresponding eigenvalues of $H$ as thick coloured lines. If the system is prepared in the state $\psi_{1}$, and the magnetic field is varied adiabatically (slowly) to take the system through the avoided crossing, the system will remain in the same eigenstate (i.e. follow the thick pink line), assuming the absence of incoherent effects such as relaxation. 


\section{The field-sweep experiment}

We now consider the density operator evolution for the procedure sketched in Fig. 2; (1) sample hydrogenation at a static field strength of $+2 \mu \mathrm{T},(2)$ an instantaneous (diabatic) field reversal to $-2 \mu \mathrm{T},(3)$ a field sweep from $-2 \mu \mathrm{T}$ to $+2 \mu \mathrm{T}$ under the adiabatic approximation.

\section{Sample hydrogenation}

Immediately after hydrogenation of the precursor with para-enriched hydrogen, the spin density operator $\rho_{0}$ is given by expressing the density operator

$$
\rho_{\text {singlet }}=\frac{1}{2}\left(\left|S_{0} \alpha\right\rangle\left\langle S_{0} \alpha|+| S_{0} \beta\right\rangle\left\langle S_{0} \beta\right|\right)
$$

in the Hamiltonian eigenbasis, and removing off-diagonal elements between non-degenerate eigenvalues. The offdiagonal elements are coherences, which are assumed to be averaged to zero during the long hydrogenation period (long in comparison to the inverse of the coherences). The result is of the form

$$
\rho_{0}=\sum_{\psi} p_{\psi}^{\mathrm{ini}}|\psi\rangle\langle\psi|
$$

where $|\psi\rangle$ is a Hamiltonian eigenstate, and $p_{\psi}^{\text {ini }}$ is the population of state $\psi$.

Since the hydrogenation field is not at an avoided crossing, the eigenstates of $H_{0}$ are close to the eigenstates of $H$. The initial populations of the $H_{0}$ eigenstates, assuming pure $\mathrm{pH}_{2}$, are

$$
\begin{aligned}
& p_{1}^{\text {ini }}=\left|\left\langle S_{0} \mid S_{0} \alpha\right\rangle^{\prime}\right|^{2}=\frac{\left(1+\sin \left(\theta_{\mathrm{G}}\right)\right)}{4} \\
& p_{2}^{\text {ini }}=\left|\left\langle S_{0} \mid T_{+1} \alpha\right\rangle^{\prime}\right|^{2}=0 \\
& p_{3}^{\text {ini }}=\left|\left\langle S_{0} \mid T_{0} \alpha\right\rangle^{\prime}\right|^{2}=\frac{\left(1-\sin \left(\theta_{\mathrm{G}}\right)\right)}{4} \\
& p_{4}^{\text {ini }}=\left|\left\langle S_{0} \mid T_{-1} \alpha\right\rangle^{\prime}\right|^{2}=0 \\
& p_{5}^{\text {ini }}=\left|\left\langle S_{0} \mid S_{0} \beta\right\rangle^{\prime}\right|^{2}=\frac{\left(1+\sin \left(\theta_{\mathrm{G}}\right)\right)}{4} \\
& p_{6}^{\text {ini }}=\left|\left\langle S_{0} \mid T_{+1} \beta\right\rangle^{\prime}\right|^{2}=0 \\
& p_{7}^{\text {ini }}=\left|\left\langle S_{0} \mid T_{0} \beta\right\rangle^{\prime}\right|^{2}=\frac{\left(1-\sin \left(\theta_{\mathrm{G}}\right)\right)}{4} \\
& p_{8}^{\text {ini }}=\left|\left\langle S_{0} \mid T_{-1} \beta\right\rangle^{\prime}\right|^{2}=0,
\end{aligned}
$$

where $\left\langle S_{0}\right|$ is shorthand for $\left(\left\langle S_{0} \alpha\right|+\left\langle S_{0} \beta\right|\right) / 2$.

\section{Diabatic field reversal}

Diabatic field reversal from $+2 \mu \mathrm{T}$ to $-2 \mu \mathrm{T}$ projects the density operator $\rho_{0}$ onto the Hamiltonian eigenbasis at this new field, and this operation has no effect on the density operator. The states $\left|S_{0} \alpha\right\rangle^{\prime}$ and $\left|S_{0} \beta\right\rangle^{\prime}$ retain their populations.

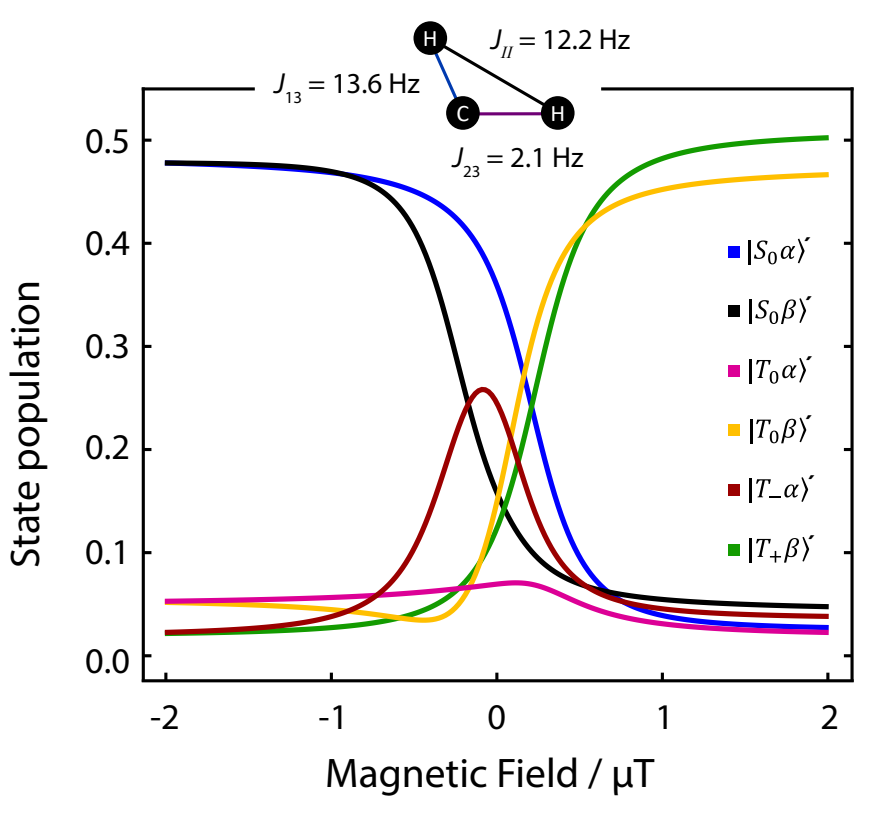

FIG. 5. State populations during an adiabatic -2 to $+2 \mu \mathrm{T}$ field sweep for the 3 -spin-1/2 system of maleic acid. The $J$ couplings are shown in the inset figure.

\section{Adiabatic field sweep}

Applying a perfectly adiabatic field sweep from $-2 \mu \mathrm{T}$ to $+2 \mu \mathrm{T}$ causes the state populations to evolve along with the Hamiltonian eigenstates. Hence, the populations of states $\left|S_{0} \alpha\right\rangle^{\prime}$ and $\left|S_{0} \beta\right\rangle^{\prime}$ are transferred into populations of states $\left|T_{+1} \beta\right\rangle^{\prime}$ and $\left|T_{0} \beta\right\rangle^{\prime}$, respectively, corresponding to ${ }^{13} \mathrm{C}|\beta\rangle$ polarization. This can be seen visually by following the thick eigenvalue lines in Fig. 4. The state populations during the field sweep are shown in Fig. 5 for two differently $J$-coupled spin systems.

In cases for which the proton singlet state is an exact Hamiltonian eigenstate, this process can give rise, in principle, to unity ${ }^{13} \mathrm{C}$ polarization. However, for $\left[1-{ }^{13} \mathrm{C}\right]$ maleic acid, the incomplete magnetic equivalence of the two protons means that the proton singlet state is not an exact eigenstate of the initial spin Hamiltonian, leading to a limit of $90 \%{ }^{13} \mathrm{C}$ polarization, neglecting relaxation and instrumental imperfections.

\section{MATERIALS AND METHODS}

For ultralow-field experiments, a magnetic shield (MS1F, Twinleaf LLC, Princeton, U.S.) was used to provide a $10^{6}$ shielding factor against external magnetic fields. Static internal magnetic fields for shimming were produced using built-in $B_{\mathrm{x}}, B_{\mathrm{y}}$, and $B_{\mathrm{z}}$ coils, powered with computer-controlled DC calibrators (Krohn-Hite, model 523, Brockton, U.S.), providing three-axis field control. Time-dependent "pulse" fields were generated with three nested Helmholtz coils wound on a 3D-printed former, 
where each coil was driven with a separate isolated power amplifier (AE Techron 7224-P, Elkhart, U.S.), with waveforms produced with the gradient controller of a low-field NMR spectrometer (Kea II, Magritek GmbH, Aachen, Germany).

NMR tubes held in the ZULF chamber and $1.4 \mathrm{~T}$ SpinSolve were connected with PEEK tubing $(1 / 16$ inch O.D., 0.02 inch I.D.), as shown in Fig. 6. Gas and liquid flow were controlled by pneumatically actuated valves (Swagelok, Solon, U.S.); the pneumatic valves were controlled via TTL outputs of the Magritek spectrometer. Sample hydrogenation was followed by shuttling into the SpinSolve by reversing the gas flow. The sample transport was performed with helium gas (any unreactive gas could be used), and took $1 \mathrm{~s}$. In order to prevent the sample from passing through any fields that could lead to undesired state-mixing during sample transport, a penetrating solenoid was used to provide a guiding field during transit out of the magnetic shield.

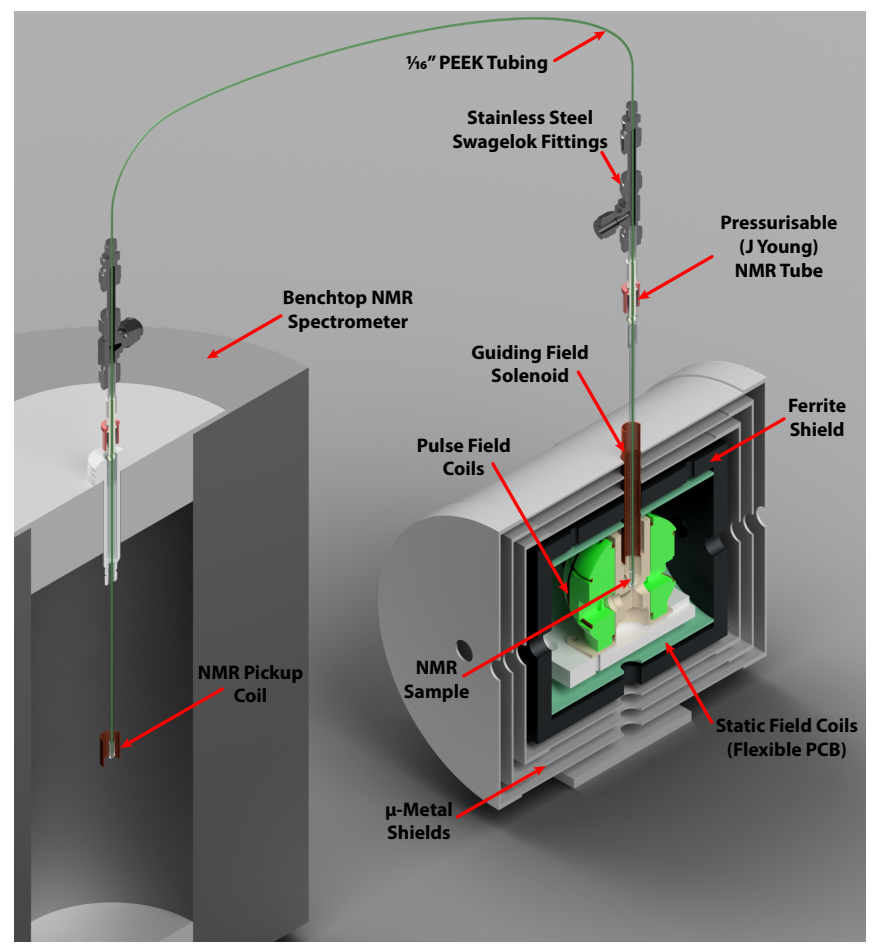

FIG. 6. Schematic of the experimental apparatus. The sample was hydrogenated in the magnetically shielded chamber, and field manipulations were performed using the pulse field coils. The sample was then pneumatically shuttled into the SpinSolve for signal acquisition.

To generate parahydrogen gas at $92 \%$ para enrichment, regular hydrogen gas (purity 99.995\%) was passed through a Bruker parahydrogen generator operating at $36 \mathrm{~K}$. Unless stated otherwise in the text, the solution prior to hydrogenation was $5 \mathrm{mM} \quad$ 1,4-bis(diphenylphosphino)butane(1,5cyclooctadiene)rhodium tetrafluoroborate and $100 \mathrm{mM}$ acetylene dicarboxylic acid in $300 \mu \mathrm{L}$ acetone.
The events sequence is shown in Fig. 2. Initially the sample is in the ZULF chamber in a $5 \mathrm{~mm}$ NMR tube, in $\mathrm{a}+10 \mu \mathrm{T}$ field provided by the pulse and guiding coils. The field was stepped down to $+2 \mu \mathrm{T}$ (chosen as a relatively low field that is still high enough for the Hamiltonian eigenstates to be, to a good approximation, the STZ' basis states) by turning off the guiding field, and parahydrogen gas was bubbled in at 5 bar for $60 \mathrm{~s}$. After a $0.5 \mathrm{~s}$ delay to allow the sample to settle, a field manipulation was applied using the pulse coils. The field manipulation shown in Fig. 2 is a sudden step to $-2 \mu \mathrm{T}$ (in $\sim 10 \mu \mathrm{s})$, followed by a linear sweep to $+2 \mu \mathrm{T}$ in $1 \mathrm{~s}$. The field was then stepped to $+2 \mu \mathrm{T}$ (or in the case shown, kept there). The guiding field was switched back on to provide a $+10 \mu \mathrm{T}$ total field, and helium gas at 6 bar was used to shuttle the sample into the SpinSolve. A $\pi / 2$ pulse was applied followed by data acquisition triggered by a TTL signal from the Kea II.

\section{RESULTS}

To evaluate the field sweep methodology we perform the hydrogenation, field sweep, and shuttling to high field procedure, and detect the ${ }^{13} \mathrm{C}$ NMR signals. We first show the effect of performing the sweep, and then study the effects of sweep direction (i.e. sweeping from -2 to $+2 \mu \mathrm{T}$ vs sweeping from +2 to $-2 \mu \mathrm{T}$ ), sweep rate, and finally provide an estimate of the achieved polarization enhancement.

\section{A. Field-sweep hyperpolarization of ${ }^{13} \mathrm{C}$ NMR signals}

To see the effect of the field sweep, a sample of acetylene dicarboxylic acid was hyperpolarized as described in the Materials and Methods section, a linear field sweep from -2 to $+2 \mu \mathrm{T}$ was performed with a duration of $1 \mathrm{~s}$, and the sample was shuttled into the SpinSolve for ${ }^{13} \mathrm{C}$ detection. For comparison, the experiment was repeated, but instead of performing the field sweep, the field was kept static at $+2 \mu \mathrm{T}$ for $1 \mathrm{~s}$. The NMR signal of the hyperpolarized sample was allowed to relax, and a thermal equilibrium spectrum was acquired. The ${ }^{13} \mathrm{C}$ NMR spectra are shown in Fig. 7. The field sweep spectrum shows a dramatic ${ }^{13} \mathrm{C}$ signal enhancement compared with thermal equilibrium where no signal is observed.

When a field sweep is not used, two relatively weak ${ }^{13} \mathrm{C}$ signals of opposite phase are observed (Fig. 7b). These signals derive from the initial singlet polarization of the protons which leads to weakly allowed transitions through singlet-triplet state mixing (see Fig. 3).

\section{B. Reversal of the field-sweep direction}

To study the influence of the field-sweep direction, the hyperpolarization procedure was repeated and the 


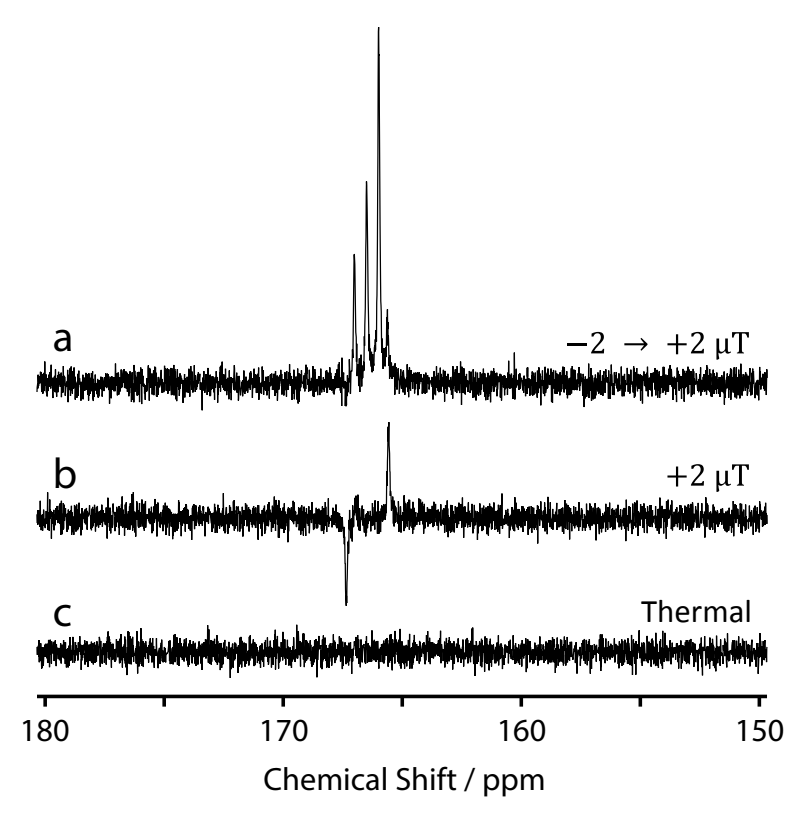

FIG. 7. Single-scan ${ }^{13} \mathrm{C}$ NMR spectra of the reaction mixture acquired without proton decoupling showing a) the signal after performing a -2 to $+2 \mu \mathrm{T}$ field sweep, b) the signal after keeping the sample in a static $+2 \mu \mathrm{T}$ field, c) the thermal equilibrium signal. The outermost spectral lines in (a) and the only visible spectral lines in (b) are caused by $S_{0} \leftrightarrow T_{0}$ state mixing (see text).

field sweep was performed in the positive direction ( -2 to $+2 \mu \mathrm{T})$ to polarize the ${ }^{13} \mathrm{C}$ spins in the $|\beta\rangle$ state, and the negative direction $(+2$ to $-2 \mu \mathrm{T})$ to polarize the ${ }^{13} \mathrm{C}$ spins in the $|\alpha\rangle$ state. The results are shown in Fig. 8. Note that helium bubbling for excess parahydrogen removal was not performed in this experiment, so the signal-tonoise is higher than in Fig. 7a.

Reversal of the sweep direction manifests as an inverted peak phase for the two spectra, and a reflection about the central resonance. This is because, in the ideal case, a positive field sweep polarizes states $\left|T_{0} \beta\right\rangle^{\prime}$ and $\left|T_{+1} \beta\right\rangle^{\prime}$, whereas a negative field sweep polarizes states $\left|T_{0} \alpha\right\rangle^{\prime}$ and $\left|T_{-1} \alpha\right\rangle^{\prime}$.

Simulations of the spectral lineshape were performed by using SpinDynamica, a package for Mathematica ${ }^{36}$. For the simulations, the density operator at the start of the field sweep was taken as $\rho_{0}$ from Eqn. 12. $\rho_{0}$ is then propagated under the time-dependent Hamiltonian as the field is swept. Proton relaxation from fluctuating external random fields (as described in Ref. ${ }^{37}$ ) is included. The correlation between the random field fluctuations at the two spin-sites was set to 0.9. The best-fit estimates of the correlation time $\tau_{c}$ and root-mean-square amplitude of the fluctuating external random fields were given by $\left(B_{\mathrm{ran}}^{\mathrm{rms}}\right)^{2} \tau_{c}=6 \times 10^{-16} \mathrm{~T}^{2} \mathrm{~s}$.

Although the sample is in precisely zero field at the centre of the sweep, it still matters whether the field is increased from zero in the positive or negative sense. This is because the quantum state in zero field depends on its preparation history, and in particular whether the zerofield state was prepared by an approach from the positive or negative field direction.

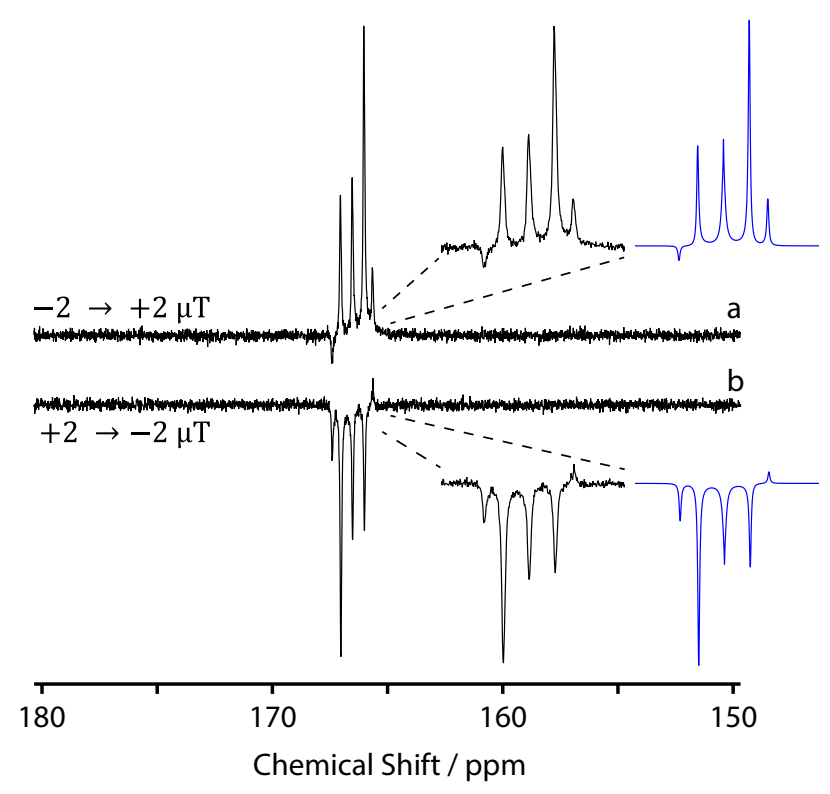

FIG. 8. Single-scan ${ }^{13} \mathrm{C}$ NMR spectra without proton decoupling showing a) the hyperpolarized $\left[1-{ }^{13} \mathrm{C}\right]$ maleic acid signal acquired after performing a -2 to $+2 \mu \mathrm{T}$ field sweep, b) the hyperpolarized sample signal acquired after performing a +2 to $-2 \mu \mathrm{T}$ field sweep. Simulations of the spectra are shown in blue.

\section{Effect of field sweep duration on polarization transfer efficiency}

To understand the effect of the field sweep duration on the degree of polarization transfer to the ${ }^{13} \mathrm{C}$ spin, the hyperpolarization step was performed on a series of reaction mixtures, and a field sweep from -2 to $+2 \mu \mathrm{T}$ was applied with a different duration for each. In Fig. 9 the ${ }^{13} \mathrm{C}$ signal intensities (as measured by peak integration) are shown, along with a SpinDynamica simulation of the ${ }^{13} \mathrm{C}$ polarization. The SpinDynamica simulations were performed with the same parameters as used for Fig. 8, but with the fluctuating external random field characterised by the parameters $\left(B_{\mathrm{ran}}^{\mathrm{rms}}\right)^{2} \tau_{c}=2 \times 10^{-16} \mathrm{~T}^{2} \mathrm{~s}$, which represents a weaker random field than that used in the simulations in Fig. 8. The reason for the discrepancy is unknown, but might reflect a variation in the oxygen content of the solutions.

For efficient polarization transfer, the adiabatic condition needs to be met. Therefore, for longer sweep durations the ${ }^{13} \mathrm{C}$ signal is larger, as the process becomes more adiabatic. This is true up to a point, but for the longest sweep durations ( $2 \mathrm{~s}$ and $5 \mathrm{~s}$ ), the signal is notably attenuated by relaxation. 


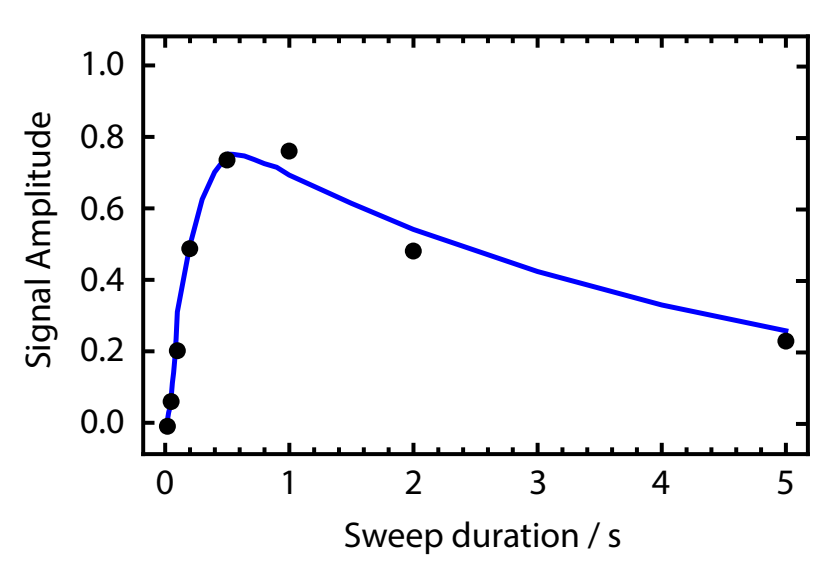

FIG. 9. Signal intensity measured for the ${ }^{13} \mathrm{C}$ spins after applying a -2 to $+2 \mu \mathrm{T}$ field sweep of variable duration, shown by black circles. A SpinDynamica simulation of the transfer efficiency is shown by the blue line. Details of the simulation are given in the text. The signal intensity is normalized to the maximum conversion amplitude of $\sim 0.9$ in the absence of relaxation.

\section{Polarization Enhancement}

To estimate the polarization level, a sample of $1 \mathrm{mM}$ [1${ }^{13} \mathrm{C}$ ] acetylene dicarboxylic acid $\left({ }^{13} \mathrm{C}\right.$-enriched) and $5 \mathrm{mM}$ rhodium catalyst in acetone was hydrogenated for $10 \mathrm{~s}$ at 1 bar parahydrogen pressure, before performing a -2 to $+2 \mu \mathrm{T}$ field sweep in $1 \mathrm{~s}$ and shuttling to the SpinSolve for detection. After letting the polarized signal decay, a 32 scan thermal spectrum was acquired on the same sample. These results are shown in Fig. 10. The $\left[1-{ }^{13} \mathrm{C}\right]$ maleic acid peak is not visible in the thermal spectrum, so the polarization was estimated by comparison to the $13.5 \mathrm{M}$ natural abundance acetone solvent peak at $207 \mathrm{ppm}$. The signal enhancement on the product [1$\left.{ }^{13} \mathrm{C}\right]$ maleic acid was estimated to be by a factor of 10,500 , corresponding to a polarization level of $>1 \%$ in the $1.4 \mathrm{~T}$ field.

\section{CONCLUSIONS}

We have presented a method to polarize the ${ }^{13} \mathrm{C}$ spin in $\left[1-{ }^{13} \mathrm{C}\right]$ maleic acid by transferring the proton singlet order, originating from the parahydrogen proton pair, using a field sweep. The field sweeps used are typically -2 to $+2 \mu \mathrm{T}$ and performed in $1 \mathrm{~s}$, although variations of this were used to evaluate the technique. This method can in theory lead to up to $100 \%{ }^{13} \mathrm{C}$ polarization, although this depends on the singlet order polarization level of the hydrogenated molecule prior to the sweep, and the degree of adiabaticity of the sweep used. We have provided a theoretical framework for understanding these field sweep experiments, which is also applicable to the more commonly used 'field cycling' method in $\mathrm{AA}^{\prime} \mathrm{X}$ spin

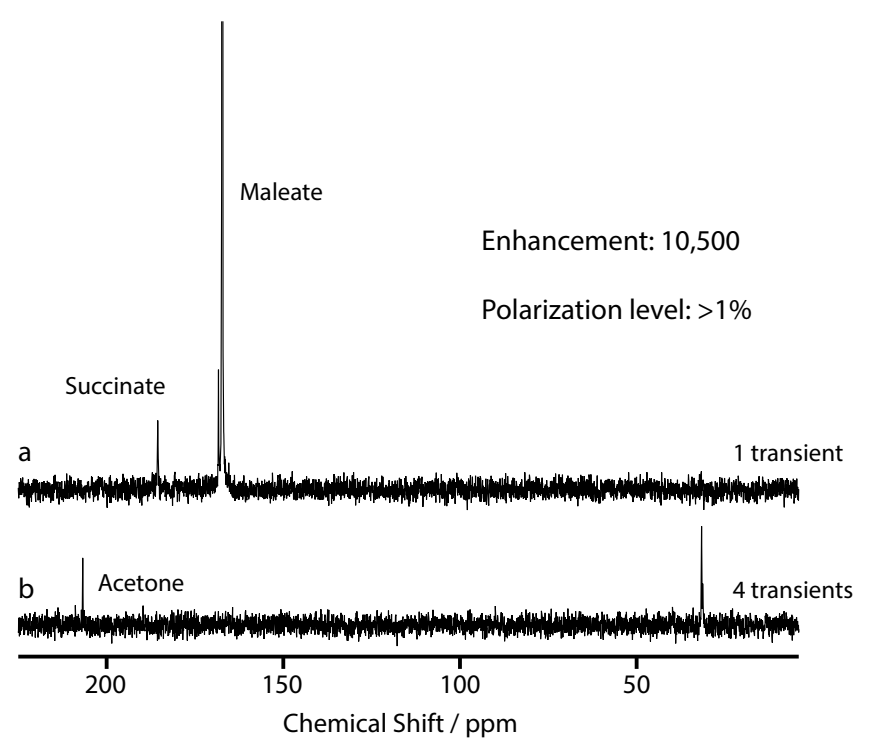

FIG. 10. Proton decoupled ${ }^{13} \mathrm{C}$ NMR spectra with $2 \mathrm{~Hz}$ line broadening showing a) hyperpolarized $\left[1-{ }^{13} \mathrm{C}\right]$ maleic acid after performing a $1 \mathrm{~s}-2$ to $+2 \mu \mathrm{T}$ field sweep, b) thermalequilibrium signal acquired with 32 transients. The product peak is not visible in the thermal equilibrium spectrum, so the signal enhancement was estimated by integral comparison to the acetone peak at $207 \mathrm{ppm}$.

systems $^{27-31}$.

We hope to combine this technique with an experiment in which the hyperpolarized metabolite [1$\left.{ }^{13} \mathrm{C}\right]$ fumarate can be produced via parahydrogen by trans-hydrogenation ${ }^{38}$. This may allow for the production of hyperpolarized metabolites for in vivo imaging in a continuous-flow manner.

The required sweep duration for adiabaticity is inversely proportional to $\left|\omega_{\text {IS }}^{\Delta}\right|$. Given the relatively large sweep range used in these experiments $(4 \mu \mathrm{T})$, this corresponds to a duration in the order of $1 \mathrm{~s}$ for $\left[1-{ }^{13} \mathrm{C}\right]$ maleic acid, in which $\omega_{\mathrm{IS}}^{\Delta}=2 \pi \times 10.5 \mathrm{~Hz}$. For $\left[1-{ }^{13} \mathrm{C}\right]$ fumaric acid, $\omega_{\text {IS }}^{\Delta}=2 \pi \times 3.4 \mathrm{~Hz}^{21}$, which means longer sweep durations will be necessary to maintain adiabaticity, and signal losses due to relaxation might become significant. For this reason, we are working to employ 'constant adiabaticity' field sweep profiles ${ }^{39}$, which will reduce the necessary field sweep duration as compared to the nonoptimal linear profiles used in this work.

\section{ACKNOWLEDGEMENTS}

This work was supported by the Engineering and Physical Sciences Research Council (grant numbers EP/N002482/1, EP/M508147/1, EP/P009980), the Royal Society, and Bruker BioSpin. This project has received funding from the European Union's Horizon 2020 research and innovation programme under the Marie Skłodowska-Curie grant agreement number 766402. J. 
E. would like to thank the Royal Society of Chemistry for a researcher mobility grant.

\section{REFERENCES}

${ }^{1}$ E. Cavallari, C. Carrera, M. Sorge, G. Bonne, A. Muchir, S. Aime, and F. Reineri, Scientific reports 8, 8366 (2018).

${ }^{2}$ J.-B. Hövener, A. N. Pravdivtsev, B. Kidd, C. R. Bowers, S. Glöggler, K. V. Kovtunov, M. Plaumann, R. Katz-Brull, K. Buckenmaier, A. Jerschow, et al., Angewandte Chemie International Edition 57, 11140 (2018).

${ }^{3}$ P. Berthault, G. Huber, and H. Desvaux, Progress in Nuclear Magnetic Resonance Spectroscopy 1, 35 (2009).

${ }^{4}$ J. H. Ardenkjær-Larsen, B. Fridlund, A. Gram, G. Hansson, L. Hansson, M. H. Lerche, R. Servin, M. Thaning, and K. Golman, Proceedings of the National Academy of Sciences 100, 10158 (2003).

${ }^{5}$ C. R. Bowers and D. P. Weitekamp, Journal of the American Chemical Society 109, 5541 (1987).

${ }^{6}$ S. Glöggler, J. Colell, and S. Appelt, Journal of Magnetic Resonance 235, 130 (2013).

${ }^{7}$ J. Natterer and J. Bargon, Progress in Nuclear Magnetic Resonance Spectroscopy 31, 293 (1997).

${ }^{8}$ S. B. Duckett and C. J. Sleigh, Progress in Nuclear Magnetic Resonance Spectroscopy 34, 71 (1999).

${ }^{9}$ R. A. Green, R. W. Adams, S. B. Duckett, R. E. Mewis, D. C. Williamson, and G. G. Green, Progress in nuclear magnetic resonance spectroscopy , 1 (2012).

${ }^{10}$ K. L. Ivanov, A. N. Pravdivtsev, A. V. Yurkovskaya, H.-M. Vieth, and R. Kaptein, Progress in Nuclear Magnetic Resonance Spectroscopy 81, 1 (2014).

${ }^{11} \mathrm{~J}$. Barkemeyer, M. Haake, and J. Bargon, Journal of the American Chemical Society 117, 2927 (1995).

${ }^{12}$ M. Haake, J. Natterer, and J. Bargon, Journal of the American Chemical Society 118, 8688 (1996).

${ }^{13}$ M. Goldman and H. Jóhannesson, Comptes Rendus Physique 6, 575 (2005).

${ }^{14}$ P. Bhattacharya, E. Y. Chekmenev, W. H. Perman, K. C. Harris, A. P. Lin, V. A. Norton, C. T. Tan, B. D. Ross, and D. P. Weitekamp, Journal of magnetic resonance 186, 150 (2007).

${ }^{15}$ E. Y. Chekmenev, V. A. Norton, D. P. Weitekamp, and P. Bhattacharya, Journal of the American Chemical Society 131, 3164 (2009).

${ }^{16}$ S. Kadlecek, K. Emami, M. Ishii, and R. Rizi, Journal of Magnetic Resonance 205, 9 (2010).

${ }^{17}$ S. Bär, T. Lange, D. Leibfritz, J. Hennig, D. von Elverfeldt, and J.-B. Hövener, Journal of Magnetic Resonance 225, 25 (2012).

${ }^{18}$ A. S. Kiryutin, K. L. Ivanov, A. V. Yurkovskaya, H.-M. Vieth, and N. N. Lukzen, Physical Chemistry Chemical Physics 15, 14248 (2013).
${ }^{19}$ M. B. Franzoni, D. Graafen, L. Buljubasich, L. Schreiber, H. W. Spiess, and K. Münnemann, Physical Chemistry Chemical Physics 15, 17233 (2013).

${ }^{20}$ C. Cai, A. M. Coffey, R. V. Shchepin, E. Y. Chekmenev, and K. W. Waddell, The Journal of Physical Chemistry B 117, 1219 (2013).

${ }^{21}$ J. Eills, G. Stevanato, C. Bengs, S. Glöggler, S. J. Elliott, J. Alonso-Valdesueiro, G. Pileio, and M. H. Levitt, Journal of Magnetic Resonance 274, 163 (2017).

${ }^{22}$ G. Stevanato, J. Eills, C. Bengs, and G. Pileio, Journal of Magnetic Resonance 277, 169 (2017).

${ }^{23}$ A. N. Pravdivtsev, A. V. Yurkovskaya, H.-M. Vieth, K. L. Ivanov, and R. Kaptein, ChemPhysChem 14, 3327 (2013).

${ }^{24}$ D. A. Barskiy, K. V. Kovtunov, I. V. Koptyug, P. He, K. A. Groome, Q. A. Best, F. Shi, B. M. Goodson, R. V. Shchepin, M. L. Truong, et al., ChemPhysChem 15, 4100 (2014).

${ }^{25}$ M. L. Truong, T. Theis, A. M. Coffey, R. V. Shchepin, K. W. Waddell, F. Shi, B. M. Goodson, W. S. Warren, and E. Y. Chekmenev, The journal of physical chemistry C 119, 8786 (2015).

${ }^{26}$ M. C. Tayler, T. F. Sjolander, A. Pines, and D. Budker, Journal of Magnetic Resonance 270, 35 (2016).

${ }^{27}$ H. Jóhannesson, O. Axelsson, and M. Karlsson, Comptes Rendus Physique 5, 315 (2004).

${ }^{28}$ M. Goldman, H. Jóhannesson, O. Axelsson, and M. Karlsson, Magnetic resonance imaging 23, 153 (2005).

${ }^{29}$ L. Buljubasich, M. Franzoni, H. W. Spiess, and K. Münnemann, Journal of Magnetic Resonance 219, 33 (2012).

${ }^{30}$ A. S. Kiryutin, A. V. Yurkovskaya, R. Kaptein, H.-M. Vieth, and K. L. Ivanov, The journal of physical chemistry letters $\mathbf{4}$, 2514 (2013).

${ }^{31}$ E. Cavallari, C. Carrera, T. Boi, S. Aime, and F. Reineri, The Journal of Physical Chemistry B 119, 10035 (2015).

${ }^{32}$ D. A. Barskiy, R. V. Shchepin, A. M. Coffey, T. Theis, W. S. Warren, B. M. Goodson, and E. Y. Chekmenev, Journal of the American Chemical Society 138, 8080 (2016).

${ }^{33}$ J. R. Lindale, S. L. Eriksson, C. P. Tanner, Z. Zhou, J. F. Colell, G. Zhang, J. Bae, E. Y. Chekmenev, T. Theis, and W. S. Warren, Nature communications 10, 395 (2019).

${ }^{34}$ M. C. Tayler, T. Theis, T. F. Sjolander, J. W. Blanchard, A. Kentner, S. Pustelny, A. Pines, and D. Budker, Review of Scientific Instruments 88, 091101 (2017).

${ }^{35}$ N. N. Lukzen and U. Steiner, Molecular Physics 86, 1271 (1995).

${ }^{36}$ C. Bengs and M. H. Levitt, Magnetic Resonance in Chemistry 56, 374 (2018).

${ }^{37} \mathrm{M}$. Carravetta and M. H. Levitt, The Journal of chemical physics 122, 214505 (2005).

${ }^{38}$ B. Ripka, J. Eills, H. Kouřilová, M. Leutzsch, M. H. Levitt, and K. Münnemann, Chemical Communications (2018).

${ }^{39}$ B. A. Rodin, K. F. Sheberstov, A. S. Kiryutin, J. T. Hill-Cousins, L. J. Brown, R. C. Brown, B. Jamain, H. Zimmermann, R. Z. Sagdeev, A. V. Yurkovskaya, et al., The Journal of chemical physics 150, 064201 (2019). 